\title{
Information search as a function of conceptual and environmental complexity'
}

\author{
PETER SUEDFELD AND SIEGFRIED STREUFERT \\ RUTGERS, THE STATE UNIVERSITY
}

Individuals operating at complex and at simple levels of conceptual structure played a tactical game for three $1 / 2-\mathrm{hr}$. periods. There was a negative relationship between information input and subsequent information search. Conceptually simple $S_{s}$, while generally requesting more information, wanted feedback about ongoing events; complex $S$ s requested information about new aspects of the game.

In a recent study (Streufert, Suedfeld, \& Driver, 1965a), it was demonstrated that active information search in a game situation differed as a function of information load (rate of information input) and of the conceptual complexity of the Ss composing the group. During playing periods where information input load was high, Ss requested further information less frequently than during low-load periods. While there was no overall difference in information search between groups of Ss differing in conceptual structure (as defined and described in Schroder, Driver, \& Streufert, in press), a significant interaction effect was found: groups composed of conceptually simple Ss were more influenced by the environment than were complex groups, in that the former showed much more information search under low load and much less under high load. Complex groups maintained a relatively stable level of search in all conditions.

The study reported here is a partial replication of Streufert et al (1965a) using individual players rather than groups; three load conditions instead of seven; and $1-1 / 2 \mathrm{hr}$. of game time as contrasted to approximately $5 \mathrm{hr}$. Besides wanting to see whether a more economical experimental procedure would yield consonant results, we were also interested in whether the tactical game which had previously been used only with groups of Ss (Streufert, Clardy, Driver, Karlins, Schroder, \& Suedfeld, 1965b) would be appropriate for use with single Ss. Last, we wished to investigate not only the amount but also the kind of information which Ss requested: the theory of Schroder etal (in press) predicts, but our previous study did not test, that simple Ss would be preoccupied with 'monitoring' information (keeping track of past and ongoing events) while complex Ss would be more interested in new plans and potentialities. Subjects

Fighteen male and 18 female undergraduates, enrolled in an introductory psychology course, were used as Ss. Half of them had been identified as conceptually complex and half as simple by the same technique as was used in the previous study, the Sentence Completion Test (Schroder \& Streufert, 1962).

\section{Procedure}

Each $\mathrm{S}$ spent $90 \mathrm{~min}$. playing the tactical game used in Streufert et al (1965a) and other previous experiments, and described in Streufert et al (1965b). Briefly, each S commanded a simulated combined-arms (land, sea, and air) force and directed the force in the invasion of an island. He made his moves by transmitting written orders to $\mathrm{E}$, who from time to time sent him written messages concerning the progress of the game. Messages were exchanged through a wall chute. In the present study, the session was divided into three 30-min. periods. During the first (warm-up) period, each $\mathbf{S}$ received 10 messages. During the second (critical) period, three levels of input load were used, with six complex and six simple Ss randomly assigned to each level:

High: 30 messages

Moderate: 10 messages

Low: 2 messages

During the third (test) period, information was provided only in response to a specific information request by $S$. Of course, Ss were not told the duration of the session, the division into periods, nor the information input pattern; at no time was there any restriction on the number of orders $S$ could originate.

Each order form handed in by $S$ included only one order. Forms were categorized as either information search orders (initiating a reconnaisance action or requesting a progress report) or tactical orders (initiating or continuing an actual operation-landing, attack, defense, etc. - in the game).

\section{Results and Discussion}

Analysis of variance for the proportion of information search moves to total orders during the test period showed both main effects to be significant (for information load during the critical period, $\mathrm{F}=20.54, \mathrm{p}<.001$; for conceptual structure, $F=5.56, p<.05$ ). The proportion of information search orders decreased as load increased; and, except in the high load condition, simple Ss requested more information than complex Ss. Conceptual structure differences were significant $(p<.05)$ in the low and the moderate load conditions, but not in the high load condition (critical difference $=10.66$; differences were $13.83,10.67$, and 3.17 , respectively).

A further analysis was performed to indicate the relative proportion of novel situations investigated by Ss during the test period. Information requests were scored according to whether they referred to an ongoing event ("How is the attack at X progressing?'") or to a situation or location where $\mathrm{S}$ had not been engaged previously 
("Send intelligence agents to report on enemy strength at $\mathrm{Y}$ "). The proportion of "novel" to total information requests was significantly higher for complex than for simple Ss $\left(\mathrm{X}^{2}=4.24, \mathrm{p}<.05\right)$.

The negative relationship between information input and subsequent information search replicated the findings of Streufert et al (1965a). The fact that simple Ss performed more information search in the low and moderate conditions was in accordance with the theory (Schroder et al, in press), because such Ss are thought to use information in a more temporally isolated fashion (see also Streufert \& Schroder, 1965). Since the individual bases only one or a few responses on each piece of information, he needs more information to maintain effective play than does the complexperson who can use each item, in combination with other items, as the basis for many moves. Reversal under high load may indicate that in this situation the simple Shas sufficient stimuli upon which to base his moves, while the complex person may still need information to develop his integrative strategy. Thus, the needs of the simple $\mathrm{S}$ are satisfied by large amounts of information per se, whereas the complex $S$ needs specific items of information regardless of total load. This hypothesis should be tested in the future by independently varying strategy-relevance and load.

Simple individuals were more interested in receiving feedback about ongoing activities, while complex Ss searched new, hitherto unexplored aspects of the situation. This difference in orientation had been expected on the basis of the theory, and lends further confirmation to the general approach to the study of information motivation which has been developed by Schroder et al (in press).

\section{References}

Schroder, H. M., \& Streufert, S. The measurement of four systems varying in level of abstractness (sentence completion method). Office of Naval Research Technical Report, 1962, No. 11.

Schroder, H. M., Driver, M. J., \& Streufert, S. Information processing systems in individuals and groups. New York: Holt, Rinehart, \& Winston, in press.

Streufert, S., \& Schroder, H. M. Conceptual structure, environmental complexity, and task performance. J. exp. Res. Pers., 1965, 1, $132-137$.

Streufert, S. Suedfeld, P., \& Driver, M. J. Conceptual complexity, information search, and information utilization. $J$. Pers. soc. Psychol, 1965a, 2, 736-740.

Streufert, S., Clardy, Mary-Anne, Driver, M. J., Karlins, M., Schroder H. M., \& Suedfeld, P. A tactical game as a method for the analysis of complex decision making behavior in individuals and social groups. Psychol. Rep., 1965b, 17, 723-729.

\section{Note}

1. This research was carried out while the first author was Visiting Assistant Professor at the University of Illinois, Urbana. The financial support of the University of Illinois Research Board, and the assistance of $R$. L. Hagen in conducting the experiment, are gratefully acknowledged. 